\title{
Exact Tuning of PID Controllers in Control Feedback Design *
}

\author{
Lorenzo Ntogramatzidis $^{\dagger} \quad$ Augusto Ferrante ${ }^{\star}$ \\ ${ }^{\dagger}$ Department of Mathematics and Statistics, \\ Curtin University, Perth WA 6845, Australia. \\ E-mail: L.Ntogramatzidis@curtin.edu.au . \\ ${ }^{\star}$ Dipartimento di Ingegneria dell'Informazione, \\ Università di Padova, via Gradenigo, 6/B - I-35131 Padova, Italy. \\ E-mail: augusto@dei.unipd.it
}

\begin{abstract}
In this paper, we introduce a range of techniques for the exact design of PID controllers for feedback control problems involving requirements on the steady-state performance and standard frequency domain specifications on the stability margins and crossover frequencies. These techniques hinge on a set of simple closed-form formulae for the explicit computation of the parameters of the controller in finite terms as functions of the specifications, and therefore they eliminate the need for graphical, heuristic or trial-and-error procedures. The relevance of this approach is $i$ ) theoretical, since a closed-form solution is provided for the design of PID-type controllers with standard frequency domain specifications; ii) computational, since the techniques presented here are readily implementable as software routines, for example using MATLAB ${ }^{\circledR}$; iii) educational, because the synthesis of the controller reduces to a simple exercise on complex numbers that can be solved with pen, paper and a scientific calculator. These techniques also appear to be very convenient within the context of adaptive control and self-tuning strategies, where the controller parameters have to be calculated on-line. Furthermore, they can be easily combined with graphical and first/second order plant approximation methods in the cases where the model of the system to be controlled is not known.
\end{abstract}

\section{Introduction}

Countless tuning methods have been proposed for PID controllers over the last seventy years. Accounting for all of them goes beyond the possibilities of this paper. We limit ourselves to noticing that many surveys and textbooks have been entirely devoted to these techniques, that differ from each other in terms of the

*This work was partially supported by the Australian Research Council (Discovery Grant DP0986577) and by the Italian Ministry for Education and Resarch (MIUR) under PRIN grant "Identification and Robust Control of Industrial Systems". 
specifications, the amount of knowledge on the model of the plant and in terms of tools exploited, see e.g. $[2,3,4,13]$ and the references therein.

Recently, renewed interest has been devoted to design techniques for PID controllers under frequency domain specifications, see e.g. [16, 12, 9, 8, 7]. In particular, much effort has been devoted to the computation of the parameters of the PID controllers that guarantee desired values of the gain/phase margins and of the crossover frequency. Specifications on the stability margins have always been extensively utilised in feedback control system design to ensure a robust control system. It is also common to encounter specifications on phase margin and gain crossover frequency, since these two parameters together often serve as a performance measure of the control system. Indeed, loosely speaking, the phase margin is related to characteristics of the response such as the peak overshoot and the resonant peak, while the gain crossover frequency is related to the rise time and the bandwidth, [6,5].

In the last fifteen years, three important sets of techniques have been proposed to deal with requirements on the phase/gain margins and on the gain crossover frequency, to the end of avoiding the trial-and-error nature of classical control methods based on Bode and Nichols diagrams. The first one is a graphical method hinging on design charts, and exploits an interpolation technique to determine the parameters of the PID controller. This method can be adapted to control problems involving different kinds of compensators, including phasecorrection networks, and can deal with specification on the stability margins, crossover frequencies and on the steady-state performance, [16]. A second important set of techniques that can handle specifications on the phase and gain margins relies on the approximation of the plants dynamics with a first (or second) order plus delay model, [7], [12]. A third numerical technique has been recently proposed in [9] in which the objective is the determination of the set of PID controllers that satisfy given requirements on the gain and phase margins, but without the flexibility required to also deal with specifications on the crossover frequencies and on the steady-state performance.

To overcome the difficulties and approximations of trial-and-error procedures on Bode and Nyquist plots, and of the three above described design methods, a unified design framework is presented in this paper for the closed-form solution of the feedback control problem with PID controllers. The problem of determining closed-form expressions for the parameters of the controller to exactly meet the aforementioned design specifications has been described as a difficult one, [7], and this in part explains the popularity of heuristic, numerical and graphical solutions to this problem, [6], [11]. In this paper, we show that this is not the case: simple closed-form formulae are easily established for the computation of the parameters of a PID controller that exactly meets specifications on the steady-state performance, stability margins and crossover frequencies, without the need to resort to approximations for the transfer function of the plant.

There are several advantages connected with the use of the methods presented here for the synthesis of PID controllers: $i$ ) unlike other analytical synthesis methods [14], steady-state performance specifications can be handled easily; Moreover, the desired phase/gain margins and crossover frequency can be achieved exactly, without the need for trial-and-error, approximations of the plant dynamics or graphical considerations; ii) a closed-form solution to the feedback control problem allows to analyse how the solution changes as a result of variations of the problem data; Moreover, the explicit formulae presented here can be exploited for the 
self-tuning of the controller; iii) very neat necessary and sufficient solvability conditions can be derived for each controller and each set of specifications considered, and reliable methods can be established to select the compensator structure to be employed depending on the specifications imposed; iv) Several important questions arising in the design of PID controllers can find a precise answer for the first time. For example, is it possible to determine in finite terms the range of phase margins that can be achieved at a particular gain crossover frequency for each type of compensator belonging to the family of PID controllers? $v$ ) The approach presented here can be used jointly with graphical considerations on Bode or Nichols diagrams to determine the set of solutions of the control problem in the case of inequality constraints; vi) The formulae presented here are straightforwardly implementable as $\mathrm{MATLAB}^{\circledR}$ routines. Furthermore, as shown in the several numerical examples throughout the paper, the calculation of the parameters of the controller is carried out via standard manipulations on complex numbers, and therefore appears to be very suitable for educational purposes; vii) The generality of this method allows more complex compensator structures to be taken into account, as well. For example, the proper versions of the PID and PD controllers will be considered as well; viii) In the case of additional degrees of freedom in the solution of the control problem, it is possible to exactly establish how to select the parameters to be chosen in order to guarantee the solvability of the problem; $i x$ ) The closed-form formulae that deliver the parameters of the PID controller as a function of the specifications only depend on the magnitude and argument of the frequency response of the system to be controlled at the desired crossover frequency. As such, this method can be used in conjunction with a graphical method based on any of the standard diagrams for the representation of the dynamics of the frequency response, e.g., the Bode, Nyquist or Nichols diagrams; $x$ ) In the case a mathematical model of the plant or a graphic representation of its frequency reponse are not available, the technique presented in this paper can be used on a first/second order plus delay approximation of the plant. The extra flexibility offered by the design method presented here consists in the fact that the formulae for the computation of the parameters are not linked to a particular structure of the plant. This means that, differently from other approaches based on such approximation, when a more accurate mathematical model is available for the model of the plant, the formulae presented here can still be used without modifications, and will deliver more reliable values for the parameters of the compensator.

This paper provides a unified and comprehensive exposition of this technique, not only for PID controllers in standard form, but also for PI and PD controllers, as well as for modified (bi-)proper PID and PD controllers with approximation of the derivative action. The basic problem considered throughout the paper consists in finding the parameters of the controller that guarantees a certain phase margin and crossover frequency, and to satisfy appropriate steady-state requirements. Standard PID controllers will be considered first. Differently from the synthesis with lead and lag networks [1], [15], here it is necessary to distinguish between two different types of steady-state requirements. In fact, due to the pole at the origin in the transfer function of the standard PID controller, it is essential to discriminate between the case where the steady-state specifications impose a constraint on the Bode gain (or integral constant) of the PID controller, and the case where the presence of the pole at the origin in the PID controller alone is sufficient to satisfy the steady-state requirements. The synthesis procedures differ significantly in these two scenarios. In the first case, three simple formulae yield the expression of the three parameters of the PID controller as a function of the phase margin and the crossover 
frequency required, [5]. In the solution of the second problem there is a degree of freedom that can be exploited to satisfy additional requirements. Here, we consider two possibilities. The first is the imposition of the ratio of the two time constants of the PID controllers, which is useful since that ratio directly affects the quality of the time response of the closed-loop system, and its assignment can avoid the situation of complex conjugate zeros in the transfer function of the PID controller, see also [10]. The second is the imposition of the gain margin, in addition to the phase margin. More precisely, in the case of specifications on both stability margins and on the gain crossover frequency, it is possible to compute the phase crossover frequency by solving a polynomial equation, and then compute the parameters of the controller in finite terms. In the case of PI and PD controllers, when the steady-state requirements sharply assign the integral constant, it is not possible to impose simultaneously the phase margin and the gain crossover frequency, except for very particular cases. However, when the use alone of a PI (or PD) controller is sufficient to guarantee the satisfaction of the steadystate specifications, the problem can be solved in closed form as described above.

\section{Problem formulation}

In this section we formulate the problem of the design of the parameters of a compensator belonging to the family of PID controllers such that different types of steady-state specifications are satisfied and such that the crossover frequency and the phase margin of the loop gain transfer function are equal to desired values $\omega_{g}$ and PM, respectively. For the sake of simplicity of exposition, only unity feedback schemes are considered here. The extension to non-unity feedback schemes does not present difficulties. Consider the following assumptions on the transfer function of the plant $G(s)$ :

(i) $G(s)$ has no poles in the open right half-plane and is is strictly proper;

(ii) The polar plot of $G(j \omega)$ for $\omega \geq 0$ intersects the unit circle and the negative real semiaxis only once (except for the intersection in the origin as $\omega \rightarrow \infty$ );

(iii) The modulus of $G(j \omega)$ is a monotonically decreasing function of $\omega$.

These are the standard assumptions that guarantee that the stability margins are both well defined, and that a positive value of these margin will guarantee stability of the closed-loop system by virtue of the Nyquist Criterion, [5, 6]. Consider compensators described by the one of the following transfer functions:

i) PID controller in standard form:

$$
C_{\mathrm{PID}}(s)=K_{p}\left(1+\frac{1}{T_{i} s}+T_{d} s\right) ;
$$

ii) Proper PID controllers:

$$
C_{\mathrm{PID}}^{\prime}(s)=K_{p}\left(1+\frac{1}{T_{i} s}+\frac{T_{d} s}{1+\tau_{d} s}\right)
$$


iii) PI controllers:

$$
C_{\mathrm{PI}}(s)=K_{p}\left(1+\frac{1}{T_{i} s}\right)
$$

iv) PD controllers in standard form:

$$
C_{\mathrm{PD}}(s)=K_{p}\left(1+T_{d} s\right)
$$

v) Proper PD controllers:

$$
C_{\mathrm{PD}}^{\prime}(s)=K_{p}\left(1+\frac{T_{d} s}{1+\tau_{d} s}\right)
$$

with $K_{p}, T_{i}, T_{d}, \tau_{d}>0$. The parameter $K_{p}$ is the proportional sensitivity constant, while $T_{i}$ and $T_{d}$ are the time constants of the integral and derivative actions, respectively. The modifications of the PID and PD controllers in (2) and (5) are usually introduced because the derivative action cannot be perfectly realised, and approximate filters must be used instead. The problem we are concerned with can be stated in precise terms as follows.

Problem 2.1 Consider the classic feedback control architecture in Figure 1, where $G(s)$ is the plant transfer function. Design a controller $C(s) \in\left\{C_{\mathrm{PID}}(s), C_{\mathrm{PID}}^{\prime}(s), C_{\mathrm{PI}}(s), C_{\mathrm{PD}}(s), C_{\mathrm{PD}}^{\prime}(s)\right\}$ such that the steady-state

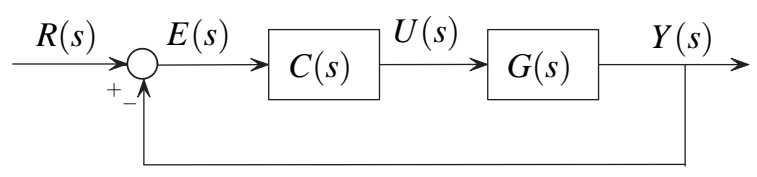

Figure 1: Unity feedback control architecture.

requirements on the tracking error $e(t) \stackrel{\text { def }}{=} r(t)-y(t)$ are satisfied, and such that the crossover frequency and the phase margin of the compensated system (loop gain transfer function) $L(s) \stackrel{\text { def }}{=} C(s) G(s)$ are $\omega_{g}$ and PM, respectively, i.e., such that

$$
\begin{aligned}
& \left|L\left(j \omega_{g}\right)\right|=1, \\
& \arg L\left(j \omega_{g}\right)=\mathrm{PM}-\pi .
\end{aligned}
$$

\section{PID controllers in standard form}

The classical PID controller is described by the transfer function:

$$
C_{\mathrm{PID}}(s)=K_{p}\left(1+\frac{1}{T_{i} s}+T_{d} s\right)=K_{p} \frac{1+T_{i} s+T_{i} T_{d} s^{2}}{T_{i} s}
$$

with $K_{p}, T_{i}, T_{d}>0$. Our aim in this section is to solve Problem 2.1 with $C(s)=C_{\mathrm{PID}}(s)$. Here, we have to discriminate between two important situations. The first is the one in which the steady-state specifications can be met by the use alone of a controller with a pole at the origin; consider e.g. the case of a type- 0 plant 
and the steady-state performance criterion of zero position error. In this case, the fact itself of using a PID controller guarantees that the steady-state requirement is satisfied. The second case of interest is the one in which the imposition of the steady-state specifications gives rise to a constraint on the Bode gain $K_{i} \stackrel{\text { def }}{=} K_{p} / T_{i}$ of $C_{\mathrm{PID}}(s)$. This situation occurs, for example, in the case of a type- 0 plant when the steady-state specification not only requires zero position error, but also that the velocity error be equal to (or smaller than) a given non-zero constant. A similar situation arises with constraints on the acceleration error for type-1 plants.

\subsection{Steady-State requirements do not constrain $K_{i}$}

First, we consider the case where the steady-state specifications do not lead to a constraint on the integral constant of the PID controller. In order to compute the parameters of the PID controller, we write $G(j \omega)$ and $C_{\mathrm{PID}}(j \omega)$ in polar form as

$$
G(j \omega)=|G(j \omega)| e^{j \arg G(j \omega)}, \quad C_{\mathrm{PID}}(j \omega)=M(\omega) e^{j \varphi(\omega)} .
$$

The loop gain frequency response can be written as $L(j \omega)=|G(j \omega)| M(\omega) e^{j(\arg G(j \omega)+\varphi(\omega))}$. If the gain crossover frequency $\omega_{g}$ and the phase margin PM of the loop gain transfer function $L(s)$ are assigned, by (6-7) it is found that $\left|L\left(j \omega_{g}\right)\right|=1$ and $\mathrm{PM}=\pi+\arg L\left(j \omega_{g}\right)$ must be satisfied. These two equations can be written as

(i) $M_{g}=1 /\left|G\left(j \omega_{g}\right)\right|$,

(ii) $\varphi_{g}=\mathrm{PM}-\pi-\arg G\left(j \omega_{g}\right)$,

where $M_{g} \stackrel{\text { def }}{=} M\left(\omega_{g}\right)$ and $\varphi_{g} \stackrel{\text { def }}{=} \varphi\left(\omega_{g}\right)$. In order to find the parameters of the controller such that $(i)$-(ii) are met, equation

$$
M_{g} e^{j \varphi_{g}}=K_{p} \frac{1+j \omega_{g} T_{i}-\omega_{g}^{2} T_{i} T_{d}}{j \omega_{g} T_{i}}
$$

must be solved in $K_{p}, T_{i}, T_{d}>0$. It is easy to see that in the solution to this problem there is a degree of freedom, since by equating the real and imaginary parts of both sides of (8) we obtain the pair of equations

$$
\begin{aligned}
& \omega_{g} M_{g} T_{i} \cos \varphi_{g}=\omega_{g} K_{p} T_{i}, \\
& -M_{g} \omega_{g} T_{i} \sin \varphi_{g}=K_{p}-K_{p} \omega_{g}^{2} T_{i} T_{d}
\end{aligned}
$$

in the three unknowns $K_{p}, T_{i}$ and $T_{d}$. A possibility to carry out the design at this point is to freely assign one of the unknowns and to solve (9-10) for the other two. However, from (9) it is easily seen that $K_{p}$ cannot be chosen arbitrarily. If we choose $T_{i}$, (9) gives $K_{p}=M_{g} \cos \varphi_{g}$, and (10) leads to

$$
T_{d}=\frac{1+\omega_{g} T_{i} \tan \varphi_{g}}{T_{i} \omega_{g}^{2}}
$$

However, in order to guarantee $K_{p}>0$ and $T_{d}>0$, the angle $\varphi_{g}$ must be such that $\cos \varphi_{g}>0$ and $T_{i}$ must be chosen to be smaller than $-1 /\left(\omega_{g} \tan \varphi_{g}\right)$. These two conditions can be simultaneously satisfied only when 
$\varphi_{g} \in(-\pi / 2,0)$. If we choose $T_{d}$, we get $K_{p}=M_{g} \cos \varphi_{g}$ and

$$
T_{i}=\frac{1}{\omega_{g}^{2} T_{d}-\omega_{g} \tan \varphi_{g}}
$$

which implies that in order to ensure $T_{i}>0$ we must choose $T_{d}$ to be greater than $\tan \varphi_{g} / \omega_{g}$. Therefore, $T_{d}$ is arbitrary when $\varphi_{g} \in(-\pi / 2,0)$, while when $\varphi_{g} \in(0, \pi / 2)$, we must choose $T_{d}>\tan \varphi_{g} / \omega_{g}$.

Another possibility to carry out the design of the PID controller in the case of unconstrained integral constant is to exploit the remaining degree of freedom so as to satisfy some further time or frequency domain requirements. There are several ways to exploit this degree of freedom in the calculation of the parameters of the controller. In the sequel, we consider two important situations: the first is the one where the ratio $T_{d} / T_{i}$ is chosen, so as to ensure, for example, that the zeros of the PID controller are real; the second is the one where a gain margin constraint is to be satisfied.

\subsubsection{Imposition of the ratio $T_{d} / T_{i}$}

A very convenient way to exploit the degree of freedom in the solution of (8) consists in the imposition of the ratio $\sigma \stackrel{\text { def }}{=} T_{d} / T_{i}$. This is convenient since it is an easily established fact that when $T_{i} \geq 4 T_{d}$, i.e., when $\sigma^{-1} \geq 4$, the zeros of the PID controller are real (and coincident when $\sigma^{-1}=4$ ), and they are complex conjugate when $\sigma^{-1}<4$. In the following theorem, necessary and sufficient conditions are given for the solvability of Problem 2.1 in the case of a standard PID controller when the ratio $\sigma$ is given. Moreover, closed-form formulae are provided for the parameters of the PID controller to meet the specifications on PM, $\omega_{g}$ and $\sigma$ exactly.

Theorem 3.1 Let $\sigma=T_{d} / T_{i}$ be assigned. Equation (8) admits solutions in $K_{p}, T_{i}, T_{d}>0$ if and only if

$$
\varphi_{g} \in\left(-\frac{\pi}{2}, \frac{\pi}{2}\right)
$$

If (11) is satsfied, the solution of (8) with $\sigma$ fixed is given by

$$
\begin{aligned}
K_{p} & =M_{g} \cos \varphi_{g} \\
T_{i} & =\frac{\tan \varphi_{g}+\sqrt{\tan ^{2} \varphi_{g}+4 \sigma}}{2 \omega_{g} \sigma} \\
T_{d} & =T_{i} \sigma
\end{aligned}
$$

Proof: (Only if). As already observed, equating real part to real part and imaginary part to imaginary part in (8) results in (9) and (10). Since $K_{p}$ must be positive, from (9) - which can be written as $K_{p}=M_{g} \cos \varphi_{g}$ - we get that $\varphi_{g}$ must satisfy $-\pi / 2<\varphi_{g}<\pi / 2$. If this inequality is satisfied, it is also easy to see that (10) always admits a positive solution. In fact, (10) can be written as the quadratic equation

$$
\omega_{g}^{2} \sigma T_{i}^{2}-\omega_{g} T_{i} \tan \varphi_{g}-1=0
$$

in $T_{i}$, that always admits two real solutions, one positive and one negative.

(If). From (12), it follows that (9) is satisfied. Moreover, since as aforementioned (10) can be writtes as (15) and $\sqrt{\tan ^{2} \varphi+4 \sigma}>|\tan \varphi|$, the positive solution is given by (13). 


\subsubsection{Imposition of the Gain Margin}

Another possibility in the solution of the control problem in the case of unconstrained $K_{i}$ is to fix the gain margin to a certain value GM. To this end, the conditions $\arg L\left(j \omega_{p}\right)=-\pi$ and $\mathrm{GM}=\left|L\left(j \omega_{p}\right)\right|^{-1}$ on the loop gain frequency response must be satisfied by definition of gain margin and phase crossover frequency $\omega_{p}$. By writing again $C_{\mathrm{PID}}(j \omega)=M(\omega) e^{j \varphi(\omega)}$, it is found that these conditions are equivalent to

$$
\begin{aligned}
M_{p} & =\frac{1}{\mathrm{GM}\left|G\left(j \omega_{p}\right)\right|}, \\
\varphi_{p} & =-\pi-\arg G\left(j \omega_{p}\right),
\end{aligned}
$$

where $M_{p} \stackrel{\text { def }}{=} M\left(\omega_{p}\right)$ and $\varphi_{p} \stackrel{\text { def }}{=} \varphi\left(\omega_{p}\right)$. Therefore, now the parameters $K_{p}, T_{i}, T_{d}>0$ of the PID controller must be determined so that $(8)$ and

$$
M_{p} e^{j \varphi_{p}}=K_{p} \frac{1+j \omega_{p} T_{i}-\omega_{p}^{2} T_{i} T_{d}}{j \omega_{p} T_{i}}
$$

are simultaneously satisfied. By equating the real and the imaginary part of (8) and (18) we obtain (9), (10) and the additional two equations

$$
\begin{aligned}
& \omega_{p} M_{p} T_{i} \cos \varphi_{p}=\omega_{p} K_{p} T_{i}, \\
& -M_{p} \omega_{p} T_{i} \sin \varphi_{p}=K_{p}-K_{p} \omega_{p}^{2} T_{i} T_{d} .
\end{aligned}
$$

From (9) and (19), we obtain the equation

$$
M_{g} \cos \varphi_{g}=M_{p} \cos \varphi_{p}
$$

in the unknown $\omega_{p}$. For the control problem to be solvable, it is required that equation (21) admits at least one strictly positive solution. At first glance, equation (21) seems transcendental in $\omega_{p}$. However, a more careful analysis reveals that the solution of (21) can be found by solving a polynomial equation in $\omega_{p}$, as the following lemma shows.

Lemma 3.1 Let $G(s)$ be a rational function in $s \in \mathbb{C}$. Then, (21) can be reduced to a polynomial equation in $\omega_{p}$. Let $n_{1}$ and $m_{1}$ represent the number of real poles/zeros of $G(s)$, and let $n_{2}$ and $m_{2}$ represent the number of complex conjugate pairs of poles/zeros of $G(s)$, respectively, and let $v \in \mathbb{Z}$ be the number of poles at the origin of $G(s)$ (with the understanding that if $v<0$, the transfer function has $-v$ zeros at the origin). Then, the degree of the polynomial equation (21) is at most

$$
\begin{cases}\max \left\{v+l, 2 m_{1}+4 m_{2}\right\} & \text { if } v \geq 0 \\ \max \left\{l,-v+2 m_{1}+4 m_{2}\right\} & \text { if } v<0\end{cases}
$$

where $l=m_{1}+m_{2}+n_{1}+n_{2}$.

Proof: Let us write the transfer function of the plant in the Bode real form:

$$
G(s)=\frac{K_{B}}{s^{v}} \frac{\prod_{i=1}^{m_{1}}\left(1+\hat{\tau}_{i} s\right) \prod_{i=1}^{m_{2}}\left(1+2 \frac{\hat{\zeta}_{i}}{\hat{\omega}_{n, i}} s+\frac{s^{2}}{\hat{\omega}_{n, i}^{2}}\right)}{\prod_{i=1}^{n_{1}}\left(1+\tau_{i} s\right) \prod_{i=1}^{n_{2}}\left(1+2 \frac{\zeta_{i}}{\omega_{n, i}} s+\frac{s^{2}}{\omega_{n, i}^{2}}\right)}
$$


with $v \in \mathbb{Z}$. In the Bode real form given above, the parameters $\tau_{i}$ and $\hat{\tau}_{i}$ are the time constants associated with the real poles/zeros, $\zeta_{i}$ and $\hat{\zeta}_{i}$ are the damping ratios and $\omega_{n, i}$ and $\hat{\omega}_{n, i}$ are the natural frequencies associated with the complex conjugate pairs of poles/zeros of $G(s)$. It follows that

$$
M_{p}=\frac{\omega_{p}^{v}}{K_{B}} \frac{\prod_{i=1}^{n_{1}} \sqrt{1+\omega_{p}^{2} \tau_{i}^{2}} \prod_{i=1}^{n_{2}} \sqrt{\left(1-\frac{\omega_{p}^{2}}{\omega_{n, i}^{2}}\right)^{2}+4 \zeta_{i}^{2} \frac{\omega_{p}^{2}}{\omega_{n, i}^{2}}}}{\mathrm{GM} \prod_{i=1}^{m_{1}} \sqrt{1+\omega_{p}^{2} \hat{\tau}_{i}^{2}} \prod_{i=1}^{m_{2}} \sqrt{\left(1-\frac{\omega_{p}^{2}}{\hat{\omega}_{n, i}^{2}}\right)^{2}+4 \hat{\zeta}_{i}^{2} \frac{\omega_{p}^{2}}{\hat{\omega}_{n, i}^{2}}}}
$$

and

$\varphi_{p}=-h \pi+\sum_{i=1}^{v}\left(-\frac{\pi}{2}\right)+\sum_{i=1}^{m_{1}} \arctan \left(\omega_{p} \hat{\tau}_{i}\right)+\sum_{i=1}^{m_{2}} \arctan \frac{2 \hat{\zeta}_{i} \omega_{p} \hat{\omega}_{n, i}}{\omega_{p}^{2}-\hat{\omega}_{n, i}^{2}}-\sum_{i=1}^{n_{1}} \arctan \left(\omega_{p} \tau_{i}\right)-\sum_{i=1}^{n_{2}} \arctan \frac{2 \zeta_{i} \omega_{p} \omega_{n, i}}{\omega_{p}^{2}-\omega_{n, i}^{2}}$

where the integer $h$ depends on the sign of $K_{B}$ and on the sign of the real parts of the second order terms of the frequency response $G(j \omega)$. As such, $\varphi_{p}$ can be written as

$$
\varphi_{p}=-(h+v-\hat{v}) \pi+\sum_{i=1}^{\hat{v}}\left(-\frac{\pi}{2}\right)+\sum_{j=0}^{l} \Phi_{j}
$$

for suitably defined angles $\Phi_{j}$, where $l=m_{1}+m_{2}+n_{1}+n_{2}$ and $\hat{v}$ is either equal to 0 when $v$ is even and equal to 1 if $v$ is odd. Therefore,

$$
\cos \varphi_{p}= \begin{cases}(-1)^{h+v-1} \cos \left(\sum_{j=0}^{l} \Phi_{j}\right) & \text { if } v \text { is even } \\ (-1)^{h+v} \sin \left(\sum_{j=0}^{l} \Phi_{j}\right) & \text { if } v \text { is odd }\end{cases}
$$

In view of the well-known formulae

$$
\begin{aligned}
& \cos \left(\sum_{j=0}^{l} \Phi_{j}\right)=\sum_{\operatorname{even} k \in\{0, \ldots, l\}}(-1)^{\frac{k}{2}} \sum_{\substack{S \subseteq\{1, \ldots l\} \\
|S|=k}}\left(\prod_{j \in S} \sin \Phi_{j} \prod_{j \notin S} \cos \Phi_{j}\right) \\
& \sin \left(\sum_{j=0}^{l} \Phi_{j}\right)=\sum_{\operatorname{odd}} k\{0, \ldots, l\} \\
&(-1)^{\frac{k}{2}} \sum_{\substack{S \subseteq\{1, \ldots l\} \\
|S|=k}}\left(\prod_{j \in S} \sin \Phi_{j} \prod_{j \notin S} \cos \Phi_{j}\right),
\end{aligned}
$$

we obtain

$$
\begin{aligned}
\cos \varphi_{p} & =\sum_{j=1}^{p}\left(\prod_{i=1}^{m_{1}} f_{i, j}^{a}\left(\arctan \left(\omega_{p} \hat{\tau}_{i}\right)\right) \prod_{i=1}^{m_{2}} f_{i, j}^{b}\left(\arctan \frac{2 \hat{\zeta}_{i} \omega_{p} \hat{\omega}_{n, i}}{\omega_{p}^{2}-\hat{\omega}_{n, i}^{2}}\right)\right. \\
& \left.\cdot \prod_{i=1}^{n_{1}} f_{i, j}^{c}\left(\arctan \left(\omega_{p} \tau_{i}\right)\right) \prod_{i=1}^{n_{2}} f_{i, j}^{d}\left(\arctan \frac{2 \zeta_{i} \omega_{p} \omega_{n, i}}{\omega_{p}^{2}-\omega_{n, i}^{2}}\right)\right)
\end{aligned}
$$

where $f_{i, j}^{k}$ (with $k \in\{a, b, c, d\}$ ) are either sine or cosine functions and $p=2^{m_{1}+m_{2}+n_{1}+n_{2}-1}$. From the goniometric identities

$$
\sin \arctan \theta=\frac{\theta}{\sqrt{1+\theta^{2}}} \quad \cos \arctan \theta=\frac{1}{\sqrt{1+\theta^{2}}}
$$


it follows that $\cos \varphi_{p}$ can be written as

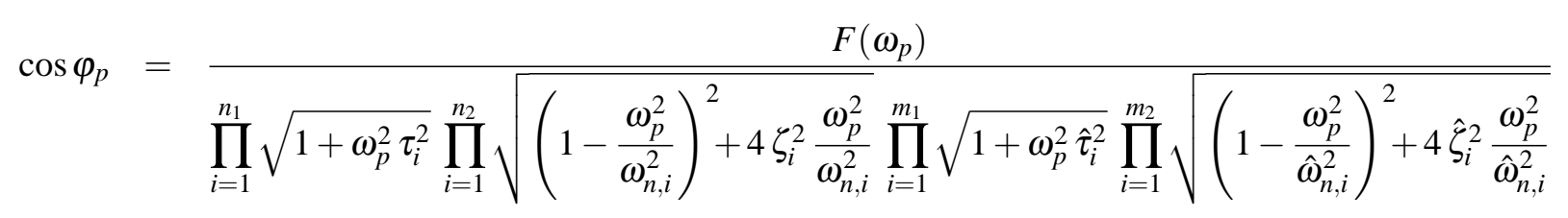

where $F\left(\omega_{p}\right)$ is a polynomial function of $\omega_{p}$ given by sums of products of the type $\omega_{p} \hat{\tau}_{i}, \omega_{p} \tau_{i}, \hat{\zeta}_{i} \omega_{p} / \hat{\omega}_{n, i}$ and $\zeta_{i} \omega_{p} / \omega_{n, i}$. Its degree depends on the term containing the largest number of sine factors in the sum (22). From (23-24) it is easy to establish that when $v$ is even,

$$
\operatorname{deg} F= \begin{cases}l & \text { if } l \text { is even } \\ l-1 & \text { if } l \text { is odd }\end{cases}
$$

and, when $v$ is odd,

$$
\operatorname{deg} F= \begin{cases}l-1 & \text { if } l \text { is even } \\ l & \text { if } l \text { is odd }\end{cases}
$$

Therefore, the degree of $F\left(\omega_{p}\right)$ is at most $l$. Hence,

$$
M_{p} \cos \varphi_{p}=\frac{\omega_{p}^{v} F\left(\omega_{p}\right)}{K_{B} \mathrm{GM} \prod_{i=1}^{m_{1}}\left(1+\omega_{p}^{2} \hat{\tau}_{i}^{2}\right) \prod_{i=1}^{m_{2}}\left[\left(1-\frac{\omega_{p}^{2}}{\hat{\omega}_{n, i}^{2}}\right)^{2}+4 \hat{\zeta}_{i}^{2} \frac{\omega_{p}^{2}}{\hat{\omega}_{n, i}^{2}}\right]}
$$

Therefore, (21) is a polynomial equation in $\omega_{p}$. In view of possible cancellations, the degree of this equation is not greater than $\max \left\{v+l, 2 m_{1}+4 m_{2}\right\}$ if $v \geq 0$ and $\max \left\{l,-v+2 m_{1}+4 m_{2}\right\}$ if $v<0$.

Remark 3.1 If the transfer function of the process is given by the product of a rational function $\hat{G}(s)$, and a delay $e^{-t_{0} s}$, i.e., if $G(s)=\hat{G}(s) e^{-t_{0} s}$, equation (21) is not polynomial in $\omega_{p}$, and it needs to be solved numerically. More details about this case are given in Section 9.

Theorem 3.2 Consider Problem 2.1 with the additional specification on the gain margin GM. Equations (8) and (18) admit solutions in $K_{p}, T_{i}, T_{d}>0$ if and only if $\varphi_{g} \in(-\pi / 2, \pi / 2)$ and (21) admits a positive solution $\omega_{p}$ such that

$$
\left\{\begin{array} { l } 
{ \omega _ { p } < \omega _ { g } } \\
{ \omega _ { g } \operatorname { t a n } \varphi _ { g } > \omega _ { p } \operatorname { t a n } \varphi _ { p } } \\
{ \omega _ { p } \operatorname { t a n } \varphi _ { g } > \omega _ { g } \operatorname { t a n } \varphi _ { p } }
\end{array} \quad \text { or } \quad \left\{\begin{array}{l}
\omega_{p}>\omega_{g} \\
\omega_{g} \tan \varphi_{g}<\omega_{p} \tan \varphi_{p} \\
\omega_{p} \tan \varphi_{g}<\omega_{g} \tan \varphi_{p}
\end{array}\right.\right.
$$

If $\varphi_{g} \in(-\pi / 2, \pi / 2)$ and (25) is satisfied, the control problem admits solutions with a PID controller, whose parameters can be computed as

$$
\begin{aligned}
K_{p} & =M_{g} \cos \varphi_{g} \\
T_{i} & =\frac{\omega_{g}^{2}-\omega_{p}^{2}}{\omega_{g} \omega_{p}\left(\omega_{p} \tan \varphi_{g}-\omega_{g} \tan \varphi_{p}\right)} \\
T_{d} & =\frac{\omega_{g} \tan \varphi_{g}-\omega_{p} \tan \varphi_{p}}{\omega_{g}^{2}-\omega_{p}^{2}}
\end{aligned}
$$


Proof: (Only if). As already seen, a necessary condition for the problem to admit solutions is that $\omega_{p}$ is a solution of (21). From (9) and (10), and from (19) and (20), we obtain

$$
\begin{aligned}
& -\omega_{g} T_{i} \tan \varphi_{g}=1-\omega_{g}^{2} T_{i} T_{d} \\
& -\omega_{p} T_{i} \tan \varphi_{p}=1-\omega_{p}^{2} T_{i} T_{d}
\end{aligned}
$$

By solving (29) and (30) in $T_{i}$ and $T_{d}$, we obtain (26-28). For $K_{p}$ to be positive, it is necessary that $\varphi_{g} \in$ $(-\pi / 2, \pi / 2)$. Moreover, the time constants $T_{i}$ and $T_{d}$ are positive if $\omega_{g}$ and $\omega_{p}$ satisfy (25).

(If). It is a matter of straighforward substitution of (26-28) into (9), (10), (19) and (20).

Remark 3.2 In view of the constraint $K_{p}>0$, (25) can be written as follows:

If $\omega_{g}>\omega_{p}$ : one of the following conditions must hold:

- $\varphi_{g}, \varphi_{p} \in\left(0, \frac{\pi}{2}\right)$ and $\varphi_{g}>\varphi_{p}$

- $\varphi_{g} \in\left(0, \frac{\pi}{2}\right)$ and $\varphi_{p} \in\left(-\frac{\pi}{2}, 0\right)$;

- $\varphi_{g}, \varphi_{p} \in\left(-\frac{\pi}{2}, 0\right)$ and $\varphi_{g}<\varphi_{p}$.

If $\omega_{g}<\omega_{p}$ : one of the following conditions must hold:

- $\varphi_{g}, \varphi_{p} \in\left(0, \frac{\pi}{2}\right)$ and $\varphi_{g}<\varphi_{p}$

- $\varphi_{g} \in\left(-\frac{\pi}{2}, 0\right)$ and $\varphi_{p} \in\left(0, \frac{\pi}{2}\right)$;

- $\varphi_{g}, \varphi_{p} \in\left(-\frac{\pi}{2}, 0\right)$ and $\varphi_{g}>\varphi_{p}$.

\subsection{Steady-State requirements constrain $K_{i}$}

Now, the Bode gain $K_{i}=K_{p} / T_{i}$ is determined via the imposition of the steady-state requirements; for example, for type-0 (resp. type-1) plants, $K_{i}$ is computed via the imposition of the velocity error (resp. acceleration error).

As such, the factor $K_{i} / s$ can be separated from $\tilde{C}_{\mathrm{PID}}(s)=1+T_{i} s+T_{i} T_{d} s^{2}$, and viewed as part of the plant. In this way, the part of the controller to be designed is $\tilde{C}_{\mathrm{PID}}(s)$, and the feedback scheme reduces to that of Figure 2. Denote $\tilde{G}(s) \stackrel{\text { def }}{=} \frac{K_{p}}{T_{i} s} G(s)$, so that the loop gain transfer function can be written as $L(s)=\tilde{C}_{\mathrm{PID}}(s) \tilde{G}(s)$. Write

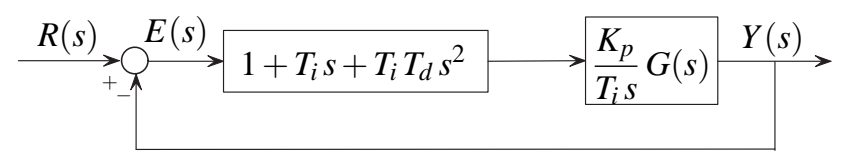

Figure 2: Modified feedback structure with unity DC gain controller.

$\tilde{G}(j \omega)$ and $\tilde{C}_{\mathrm{PID}}(j \omega)$ in polar form as

$$
\tilde{G}(j \omega)=|\tilde{G}(j \omega)| e^{j \arg \tilde{G}(j \omega)}, \quad \tilde{C}_{\mathrm{PID}}(j \omega)=M(\omega) e^{j \varphi(\omega)},
$$


so that the loop gain frequency response can be written as $L(j \omega)=|\tilde{G}(j \omega)| M(\omega) e^{j(\arg \tilde{G}(j \omega)+\varphi(\omega))}$. If the crossover frequency $\omega_{g}$ and the phase margin PM of the loop gain transfer function $L(s)$ are assigned, the equations $\left|L\left(j \omega_{g}\right)\right|=1$ and $\mathrm{PM}=\pi+\arg L\left(j \omega_{g}\right)$ must be satisfied. These can be written as

(i) $M_{g}=1 /\left|\tilde{G}\left(j \omega_{g}\right)\right|$,

(ii) $\varphi_{g}=\mathrm{PM}-\pi-\arg \tilde{G}\left(j \omega_{g}\right)$,

where $M_{g} \stackrel{\text { def }}{=} M\left(\omega_{g}\right)$ and $\varphi_{g} \stackrel{\text { def }}{=} \varphi\left(\omega_{g}\right)$. Alternatively, $M_{g}$ and $\varphi_{g}$ can be computed as functions of the frequency response of $G(s)$ at $\omega=\omega_{g}$ :

$$
\begin{aligned}
M_{g} & =\left|\frac{K_{p}}{T_{i} j \omega_{g}} G\left(j \omega_{g}\right)\right|^{-1}=\frac{\omega_{g}}{K_{i}\left|G\left(j \omega_{g}\right)\right|} \\
\varphi_{g} & =\mathrm{PM}-\pi-\arg \left[\frac{K_{p}}{T_{i} j \omega_{g}} G\left(j \omega_{g}\right)\right] \\
& =\mathrm{PM}-\frac{\pi}{2}-\arg G\left(j \omega_{g}\right),
\end{aligned}
$$

since $K_{p}, T_{i}>0$. In order to find the parameters of the controller such that (i)-(ii) are met, equation

$$
M_{g} e^{j \varphi_{g}}=1+j \omega_{g} T_{i}-T_{i} T_{d} \omega_{g}^{2}
$$

must be solved in $T_{i}>0$ and $T_{d}>0$. The closed-form solution to this problem is given in the following theorem.

Theorem 3.3 Equation (33) admits solutions in $T_{i}>0$ and $T_{d}>0$ if and only if

$$
0<\varphi_{g}<\pi \quad \text { and } \quad M_{g} \cos \varphi_{g}<1
$$

If (34) are satisfied, the solution of (33) is given by

$$
\begin{aligned}
K_{p} & =K_{i} \frac{1}{\omega_{g}} M_{g} \sin \varphi_{g} \\
T_{i} & =\frac{1}{\omega_{g}} M_{g} \sin \varphi_{g} \\
T_{d} & =\frac{1-M_{g} \cos \varphi_{g}}{\omega_{g} M_{g} \sin \varphi_{g}} .
\end{aligned}
$$

The two conditions (34) can be alternatively written as

$$
\begin{array}{cc}
\varphi_{g} \in\left(\arccos \frac{1}{M_{g}}, \pi\right) & \text { if } M_{g}>1, \\
\varphi_{g} \in(0, \pi) & \text { if } M_{g}<1,
\end{array}
$$

In fact, when $\varphi_{g} \in(0, \pi / 2)$, condition $\cos \varphi_{g}<1 / M_{g}$ is always satisfied when $M_{g}<1$, and is satisfied when $\varphi_{g}>\arccos \left(1 / M_{g}\right)$ when $M_{g}>1$. When $\varphi_{g} \in(\pi / 2, \pi)$, the condition $\cos \varphi_{g}<1 / M_{g}$ is always satisfied since $\cos \varphi_{g}<0$ and $\left(1 / M_{g}\right)>0$. As a consequence, we have the following. 
Corollary 3.1 When the ratio $K_{p} / T_{i}$ is assigned, Problem 2.1 admits solutions if and only if

- $\arg G\left(j \omega_{g}\right) \in\left(P M-\frac{3}{2} \pi, P M-\frac{\pi}{2}\right)$ if $\left|G\left(j \omega_{g}\right)\right|>\frac{\omega_{g}}{K_{i}} ;$

- $\arg G\left(j \omega_{g}\right) \in\left(P M-\frac{3}{2} \pi, P M-\frac{\pi}{2}-\arccos \frac{K_{i}\left|G\left(j \omega_{g}\right)\right|}{\omega_{g}}\right)$ if $\left|G\left(j \omega_{g}\right)\right|<\frac{\omega_{g}}{K_{i}}$.

When the steady-state specifications lead to a constraint on the ratio $K_{i}=K_{p} / T_{i}$, there are no degrees of freedom that can be exploited to assign $\sigma=T_{d} / T_{i}$. As a result, in this case the design technique based on the imposition of the phase margin and crossover frequency can lead to PID controllers with either real or complex conjugate zeros. However, in standard practice it is desirable to work with PID controllers with real zeros. The following theorem establishes necessary and sufficient conditions on the problem data and specifications for the zeros of the PID controller to be real.

Theorem 3.4 The zeros of the PID controller are real if and only if

- $\left|G\left(j \omega_{g}\right)\right|<\frac{\omega_{g}}{K_{i}}$

- $\arg G\left(j \omega_{g}\right) \in\left(P M-\frac{\pi}{2}-\arccos \frac{2 K_{i}\left|G\left(j \omega_{g}\right)\right|-\omega_{g}}{\omega_{g}}, P M-\frac{\pi}{2}\right)$.

Proof: The discriminant $T_{i}\left(T_{i}-4 T_{d}\right)$ of the numerator of $C_{\mathrm{PID}}(s)$ is greater or equal to zero if and only if $T_{i} \geq 4 T_{d}$. Using (36-37), this inequality becomes

$$
\frac{1}{\omega_{g}} M_{g} \sin \varphi_{g} \geq 4 \frac{1}{\omega_{g}} \frac{1-M_{g} \cos \varphi_{g}}{M_{g} \sin \varphi_{g}}
$$

which leads to

$$
M_{g}^{2} \cos ^{2} \varphi_{g}-4 M_{g} \cos \varphi_{g}+\left(4-M_{g}^{2}\right) \leq 0
$$

Solving (38) in $\cos \varphi_{g}$ yields

$$
\frac{2-M_{g}}{M_{g}} \leq \cos \varphi_{g} \leq \frac{2+M_{g}}{M_{g}} .
$$

However, $\frac{2+M_{g}}{M_{g}}>1$. Moreover, $\frac{2-M_{g}}{M_{g}}>-1$ and $\frac{2-M_{g}}{M_{g}}<1$ if and only if $M_{g}>1$. Hence,

$$
\left[\frac{2-M_{g}}{M_{g}}, \frac{2+M_{g}}{M_{g}}\right] \cap[-1,1] \neq \emptyset
$$

only when $M_{g}>1$. Hence, when $M_{g}<1$ the inequality (38) is never satisfied, while when $M_{g}>1$, (38) holds if and only if $\cos \varphi_{g}>\frac{2-M_{g}}{M_{g}}$. It follows that the zeros of the PID controller are real if and only if

$$
M_{g}>1 \quad \text { and } \quad 0<\varphi_{g}<\arccos \frac{2-M_{g}}{M_{g}} .
$$

The result is then a matter of substitution of the definitions of $M_{g}$ and $\varphi_{g}$ in (39). 


\section{Proper PID controllers}

The transfer function of the PID controller in standard form is not proper. To obtain a proper approximation of $C_{\mathrm{PID}}(s)$, usually a further real pole is introduced in the derivative term:

$$
\begin{aligned}
C_{\mathrm{PID}}^{\prime}(s) & =K_{p}\left(1+\frac{1}{T_{i} s}+\frac{T_{d} s}{1+\tau_{d} s}\right) \\
& =\frac{K_{p}}{T_{i} s} \frac{T_{i}\left(T_{d}+\tau_{d}\right) s^{2}+\left(T_{i}+\tau_{d}\right) s+1}{1+\tau_{d} s}
\end{aligned}
$$

with $K_{p}, T_{i}, T_{d}, \tau_{d}>0$. Due to the additional pole $s=-1 / \tau_{d}$, the transfer function $C_{\mathrm{PID}}^{\prime}(s)$ is now (bi-)proper. Typically, to obtain a good approximation of the derivative action, the frequency associated with the further pole is chosen to be much higher than the frequencies of all other poles and zeros of the loop gain transfer function. As such, the parameter $\tau_{d}$ is usually very small. Our aim here is to solve Problem 2.1 using a controller $C(s)=C_{\mathrm{PID}}^{\prime}(s)$. For the sake of brevity, we only consider the case where the steady-state specifications lead to an assignment of the Bode gain $K_{p} / T_{i}$. By defining

$$
\tilde{C}_{\mathrm{PID}}^{\prime}(s)=\frac{T_{i}\left(T_{d}+\tau_{d}\right) s^{2}+\left(T_{i}+\tau_{d}\right) s+1}{1+\tau_{d} s}
$$

and $L(s)=\tilde{C}_{\mathrm{PID}}^{\prime}(s) \tilde{G}(s)$, and by expressing $\tilde{G}(j \omega)$ and $\tilde{C}_{\mathrm{PID}}^{\prime}(j \omega)$ in polar form, $M_{g}$ and $\varphi_{g}$ can be defined as in (31) and (32). Then, in order to find the parameters of the controller, equation

$$
M_{g} e^{j \varphi_{g}}=\frac{-T_{i}\left(T_{d}+\tau_{d}\right) \omega_{g}^{2}+j\left(T_{i}+\tau_{d}\right) \omega_{g}+1}{1+j \omega_{g} \tau_{d}}
$$

must be solved in $T_{i}>0$ and $T_{d}>0$. The closed-form solution to this problem is given in the following theorem.

Theorem 4.1 Equation (41) admits solutions in $T_{i}>0$ and $T_{d}>0$ if and only if

$$
\tau_{d}<\frac{M_{g} \sin \varphi_{g}}{\omega_{g}\left(1-\cos \varphi_{g}\right)}
$$

If (42) is satisfied, the solution of (41) is given by

$$
\begin{aligned}
K_{p} & =\frac{K_{i} M_{g} \sin \varphi_{g}}{\omega_{g}}-\tau_{d}\left(1-M_{g} \cos \varphi_{g}\right) \\
T_{i} & =\frac{M_{g} \sin \varphi_{g}}{\omega_{g}}-K_{i} \tau_{d}\left(1-M_{g} \cos \varphi_{g}\right) \\
T_{d} & =\frac{1+\omega_{g}^{2} \tau_{d}^{2}}{\omega_{g}\left(\frac{M_{g} \sin \varphi_{g}}{1-M_{g} \cos \varphi_{g}}-\omega_{g} \tau_{d}\right)}
\end{aligned}
$$

Proof: It is easy to see that

$$
\begin{aligned}
& \tilde{C}_{\mathrm{PID}}(j \omega)= \\
& \frac{\left[-T_{i}\left(T_{d}+\tau_{d}\right) \omega_{g}^{2}+j\left(T_{i}+\tau_{d}\right) \omega_{g}+1\right]\left(1-j \omega_{g} \tau_{d}\right)}{\left(1+j \omega_{g} \tau_{d}\right)\left(1-j \omega_{g} \tau_{d}\right)}= \\
& \frac{-T_{i} T_{d} \omega^{2}+j \omega, T_{i}+1+j \omega^{3} \tau_{d} T_{i} T_{d}+j \omega^{3} T_{i} \tau_{d}^{2}+\omega^{2} \tau_{d}^{2}}{1+\omega^{2} \tau_{d}^{2}} .
\end{aligned}
$$


Hence, by (41) we get

$$
\begin{aligned}
M_{g} \cos \varphi_{g}\left(1+\omega_{g}^{2} \tau_{d}^{2}\right) & =-T_{i} T_{d} \omega_{g}^{2}+1+\omega_{g}^{2} \tau_{d}^{2}, \\
M_{g} \sin \varphi_{g}\left(1+\omega_{g}^{2} \tau_{d}^{2}\right) & =\omega_{g} T_{i}+\omega_{g}^{3} \tau_{d} T_{i} T_{d}+\omega_{g}^{3} T_{i} \tau_{d}^{2} .
\end{aligned}
$$

Solving both equations in $T_{i}$ and equating the results yield

$$
\frac{\left(1-M_{g} \cos \varphi_{g}\right)\left(1+\omega_{g}^{2} \tau_{d}^{2}\right)}{T_{d} \omega_{g}^{2}}=\frac{M_{g} \sin \varphi_{g}\left(1+\omega_{g}^{2} \tau_{d}^{2}\right)}{\omega_{g}\left(1+\omega_{g}^{2} \tau_{d} T_{d}+\omega_{g}^{2} \tau_{d}^{2}\right)},
$$

that can be solved to get $T_{d}$. This gives (45).

Finally, the proportional sensitivity $K_{p}$ can be computed from the ratio $K_{p} / T_{i}$ using the value of $T_{i}$ thus found.

Example 4.1 Consider the plant described by the transfer function

$$
G(s)=\frac{1}{s(s+2)},
$$

and consider the problem of determining the parameters of a PID controller that exactly achieves a phase margin of $45^{\circ}$ and gain crossover frequency $\omega_{g}=30 \mathrm{rad} / \mathrm{s}$ in the two situations:

- the acceleration error is equal to 0.005 .

- the velocity error is equal to zero; first use the remaining degree of freedom to assign the ratio $T_{i} / T_{d}=16$, and then to impose a gain margin equal to 3 .

Consider the first problem. The expression of the acceleration error is

$$
e_{\infty}^{a}=\frac{1}{\lim _{s \rightarrow 0} s^{2} L(s)}=\frac{1}{\lim _{s \rightarrow 0} s^{2} K_{p} \frac{1+T_{i} s+T_{i} T_{d} s^{2}}{T_{i} s^{2}(s+2)}}=\frac{2 T_{i}}{K_{p}} .
$$

Then, $e_{\infty}^{a}=0.005$ implies $K_{i}=K_{p} / T_{i}=400$. Define $\tilde{G}(s)=K_{i} G(s) / s$. Compute $M_{g}=30 / K_{i}|G(30 j)|=$ $9 \sqrt{904} / 4$ and $\varphi_{g}=\mathrm{PM}-\pi / 2-\arg G(30 j)=\pi / 4+\arctan 15$. Using Theorem 3.3, it is seen that the time constants of the PID controller are

$$
T_{i}=\frac{M_{g} \sin \varphi_{g}}{30}=\frac{12}{5 \sqrt{2}}, T_{d}=\frac{1-M_{g} \cos \varphi_{g}}{30 M_{g} \sin \varphi_{g}}=\frac{\sqrt{2}+63}{2160} .
$$

The zeros of the PID controller are real since $T_{i}$ is greater than $4 T_{d}$. From the ratio $K_{p} / T_{i}=400$, we find $K_{p}=400 T_{i}=960 / \sqrt{2}$. The transfer function of the PID controller is

$$
C_{\mathrm{PID}}(s)=\frac{960}{\sqrt{2}}\left(1+\frac{5 \sqrt{2}}{12 s}+\frac{\sqrt{2}+63}{2160} s\right) .
$$

Let us now solve the same problem with a PID controller with an additional pole as in (40). We first need to choose $\tau_{d}$ to be smaller than the quantity

$$
\frac{M_{g} \sin \varphi_{g}}{\omega_{g}\left(1-M_{g} \cos \varphi_{g}\right)} \simeq 0.0373
$$



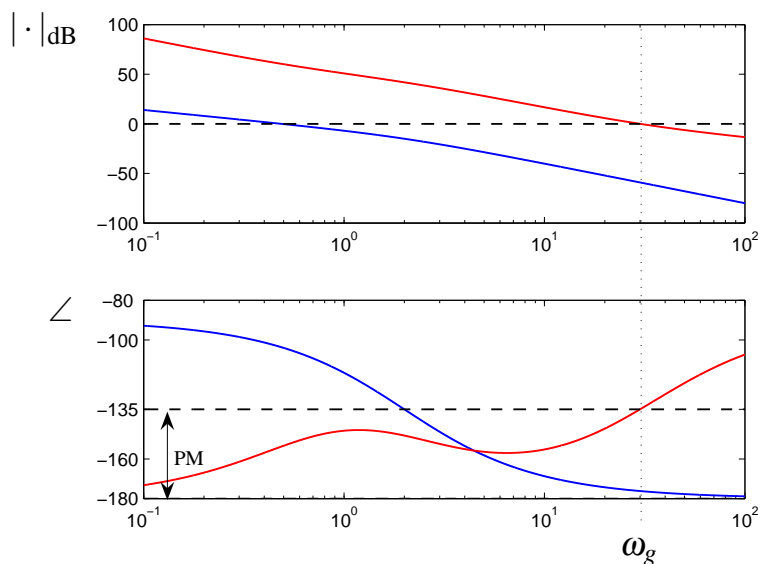

Figure 3: Bode diagram of the plant $G$ (blue solid line) and of the loop gain transfer function $L$ (red solid line).

Hence, by choosing $\tau_{d}=0.01 \mathrm{~s}$, after standard goniometric manipulations we find

$$
\begin{aligned}
T_{i} & =\frac{M_{g} \sin \varphi_{g}}{30}-\tau_{d}\left(1-M_{g} \cos \varphi_{g}\right)=\frac{177-\sqrt{2}}{100 \sqrt{2}}, \\
T_{d} & =\frac{1+30^{2} \tau_{d}^{2}}{30\left(\frac{M_{g} \sin \varphi_{g}}{1-M_{g} \cos \varphi_{g}}-30 \tau_{d}\right)}=\frac{109(63+\sqrt{2})}{300(531-3 \sqrt{2})} .
\end{aligned}
$$

From the factor $K_{p} / T_{i}=400$, we find $K_{p}=400 T_{i}=2(177 \sqrt{2}-2)$. The zeros of this controller are still real. Now let us consider the second problem, where the ratio $K_{i}=K_{p} / T_{i}$ is not constrained. Now, the pole at the origin alone guarantees that the velocity error be equal to zero. We first consider the situation where $T_{i} / T_{d}=\sigma^{-1}=16$. First, we compute $M_{g}=1 /|G(30 j)|=30 \sqrt{904}$ and $\varphi_{g}=\mathrm{PM}-\pi-\arg G(30 j)=$ $\pi / 4-\pi+\pi / 2+\arctan 15=-\pi / 4+\arctan 15$. Using (12) we find

$$
K_{p}=30 \sqrt{904} \cos \left(-\frac{\pi}{4}+\arctan 15\right)=480 \sqrt{2} .
$$

Moreover, a simple computation shows that $\tan \varphi_{g}=7 / 8$, so that using the results in Theorem 3.1, and in particular (13) with $\sigma^{-1}=16$, we find

$$
T_{i}=\frac{7+\sqrt{65}}{30} \quad \text { and } \quad T_{d}=\frac{7+\sqrt{65}}{480} .
$$

The transfer function of the PID controller in this case is

$$
C_{\mathrm{PID}}(s)=480 \sqrt{2}\left(1+\frac{30}{(7+\sqrt{65}) s}+\frac{7+\sqrt{65}}{480} s\right) .
$$

In Figure 4, the step responses of the closed-loop obtained with different values of $\sigma$ are presented. The blue line corresponds to $\sigma^{-1}=1$; the green, red and light blue ones correspond to $\sigma^{-1}=4, \sigma^{-1}=8$ and $\sigma^{-1}=24$, respectively. Hence, choosing a great value for $\sigma^{-1}$ enables oscillations and overshoot in the step response to be reduced, and has the further advantage of reducing the time constant of the derivative action $T_{d}$. Now we 


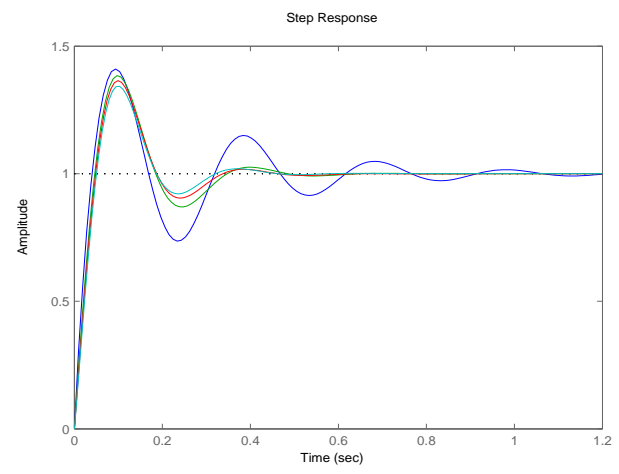

Figure 4: Unit step responses for different values of $\sigma$.

solve the same problem by imposing a gain margin equal to 3 . We compute $M_{p}$ and $\varphi_{p}$ as functions of $\omega_{p}$ :

$$
\begin{aligned}
& M_{p}=\frac{1}{\mathrm{GM}\left|G\left(j \omega_{p}\right)\right|}=\frac{\omega_{p} \sqrt{\omega_{p}^{2}+4}}{3} \\
& \varphi_{p}=-\pi-\arg G\left(j \omega_{p}\right)=-\frac{\pi}{2}+\arctan \frac{\omega_{p}}{2} .
\end{aligned}
$$

Using these expression, (21) can be written as

$$
\frac{\omega_{p} \sqrt{\omega_{p}^{2}+4}}{3} \sin \left(\arctan \frac{\omega_{p}}{2}\right)=M_{g} \cos \varphi_{g},
$$

whose unique real solution is $\omega_{p}=\sqrt{3 M_{g} \cos \varphi_{g}}>0$. Using the expressions for $M_{g}$ and $\varphi_{g}$ it is easily found that $\omega_{p}=\sqrt{720 \sqrt{8}} \mathrm{rad} / \mathrm{s}$. Hence,

$$
\varphi_{p}=-\frac{\pi}{2}+\arctan \frac{\sqrt{720 \sqrt{8}}}{2}=-\frac{\pi}{2}+\arctan \sqrt{180 \sqrt{8}}
$$

which gives $\tan \varphi_{p}=-1 / \sqrt{180 \sqrt{8}}$. It is easily verified that the conditions in Theorem 3.2 are not satisfied, since $\omega_{p}>\omega_{g}$ but $\omega_{g} \tan \varphi_{g}>\omega_{p} \tan \varphi_{p}=-2$. Let us consider the same problem with $\mathrm{PM}=\frac{2 \pi}{3}, \omega_{g}=$ $3 \mathrm{rad} / \mathrm{s}$, and $\mathrm{GM}=3$. In this case, we find $M_{g}=3 \sqrt{13}, \varphi_{g}=\frac{\pi}{6}+\arctan \frac{3}{2}$, and consequently $\tan \varphi_{g}=(2+$ $3 \sqrt{3}) /(2 \sqrt{3}-3)$. Using these values in (21) yields

$$
\omega_{p}=\sqrt{3 M_{g} \cos \varphi_{g}}=\sqrt{\frac{9}{2}(2 \sqrt{3}-3)} .
$$

As such,

$$
\varphi_{p}=-\frac{\pi}{2}+\arctan \sqrt{\frac{9(2 \sqrt{3}-3)}{8}},
$$

which yields $\tan \varphi_{p}=-1 / \sqrt{\frac{9}{8}(2 \sqrt{3}-3)}$. In this case, the conditions in Theorem 3.2 are satisfied, and the 
parameters of the PID controller can be computed in closed form as

$$
\begin{aligned}
K_{p} & =M_{g} \cos \varphi_{g}=\frac{3}{2}(2 \sqrt{3}-3) \\
T_{i} & =\frac{\omega_{g}^{2}-\omega_{p}^{2}}{\omega_{g} \omega_{p}\left(\omega_{p} \tan \varphi_{g}-\omega_{g} \tan \varphi_{p}\right)}=\frac{5-2 \sqrt{3}}{10+9 \sqrt{3}} \\
T_{d} & =\frac{\omega_{g} \tan \varphi_{g}-\omega_{p} \tan \varphi_{p}}{\omega_{g}^{2}-\omega_{p}^{2}}=\frac{26 \sqrt{3}}{144 \sqrt{3}-243} .
\end{aligned}
$$

Since $T_{i}<4 T_{d}$, the zeros of the PID controller are complex conjugate.

Example 4.2 Consider the example in [16], where a PID controller must be used for the plant

$$
G(s)=\frac{3}{s\left(s^{2}+4 s+5\right)}
$$

to achieve the following control objectives: $i$ ) acceleration constant $K_{a}=2$; ii) gain crossover frequency $\omega_{g}=$ $2.5 \mathrm{rad} / \mathrm{s}$ and phase margin $\mathrm{PM}=48^{\circ}$. First, the steady-state specification fixes the ratio $K_{i}=K_{p} / T_{i}$, as

$$
K_{a}=\lim _{s \rightarrow 0}\left(s^{2} C_{\mathrm{PID}}(s) G(s)\right)=\frac{3}{5} \frac{K_{p}}{T_{i}}=2,
$$

which in turn implies $K_{i}=K_{p} / T_{i}=10 / 3$. From $G\left(\frac{5}{2} j\right)=24 /(-25 j-200)$, we get

$$
\begin{aligned}
M_{g} & =\frac{\frac{5}{2}}{K_{i}\left|G\left(\frac{5}{2} j\right)\right|}=\frac{25 \sqrt{65}}{32} \\
\varphi_{g} & =\mathrm{PM}-\frac{\pi}{2}-\arg G\left(\frac{5}{2} j\right)=-\frac{37}{30} \pi+\arctan \frac{1}{8} .
\end{aligned}
$$

From $\sin \left(\frac{37}{30} \pi\right)=-\sin \left(\frac{7}{30} \pi\right)=-k_{1} / 8$ and $\cos \left(\frac{37}{30} \pi\right)=-\cos \left(\frac{7}{30} \pi\right)=-k_{2} / 4$, with

$$
\begin{aligned}
& k_{1}=1-\sqrt{5}+\sqrt{30+6 \sqrt{5}} \\
& k_{2}=\sqrt{7-\sqrt{5}+\sqrt{6(5-\sqrt{5})}}
\end{aligned}
$$

we obtain

$$
\begin{aligned}
& \sin \varphi_{g}=\frac{1}{\sqrt{65}}\left(k_{1}-\frac{k_{2}}{4}\right), \\
& \cos \varphi_{g}=-\frac{1}{\sqrt{65}}\left(2 k_{2}+\frac{k_{1}}{8}\right) .
\end{aligned}
$$

Plugging these values into (36-37), we obtain closed-form expressions for all the parameters of the PID controller

$$
\begin{aligned}
K_{p} & =\frac{25}{24}\left(k_{1}-\frac{k_{2}}{4}\right), \\
T_{i} & =\frac{5}{16}\left(k_{1}-\frac{k_{2}}{4}\right), \\
T_{d} & =\frac{256+25 k_{1}+400 k_{2}}{500 k_{1}-125 k_{2}} .
\end{aligned}
$$


Notice that, differently from [16], the solution provided here is given in finite terms. This ensures that the desired crossover frequency and the desired phase margin are achieved exactly.

Let us now consider the same plant, for which a PID controller must be designed to achieve a gain crossover frequency crossover frequency $\omega_{g}=1 \mathrm{rad} / \mathrm{s}$, a phase margin $\mathrm{PM}=30^{\circ}$, and a gain margin $\mathrm{GM}=3$. It is easily seen that $M_{g}=4 \sqrt{2} / 3$ and $\varphi_{g}=-\pi / 12$, so that $\tan \left(\varphi_{g}\right)=-(\sqrt{2}-\sqrt{6}) / 4$. It is easily seen that (21) admits a positive solution

$$
\omega_{p}=\sqrt{\frac{3 \mathrm{GM}}{4} M_{g} \cos \varphi_{g}}=\sqrt{3 \frac{\sqrt{3}+1}{2}} .
$$

Therefore,

$$
\varphi_{p}=-\frac{\pi}{2}+\arctan \frac{4 \omega_{p}}{5-\omega_{p}^{2}}
$$

Since $\omega_{p}<\sqrt{5}$, we find that

$$
\tan \varphi_{p}=\frac{3 \sqrt{3}-7}{4 \sqrt{6(\sqrt{3}+1)}}
$$

A simple computation yields

$$
\begin{aligned}
K_{p} & =\frac{2 \sqrt{3}+2}{3} \\
T_{i} & =\frac{4(1+3 \sqrt{3})}{15 \sqrt{3}-19}, \\
T_{d} & =\frac{9-5 \sqrt{3}}{4(1+3 \sqrt{3})} .
\end{aligned}
$$

In this case, $T_{i}>4 T_{d}$; the zeros of the PID controller are real and strictly negative.

\section{PI controllers}

The synthesis techniques presented in the previous sections can be adapted to the design of PI controllers for the imposition of phase margin and crossover frequency of the loop gain transfer function. This can be done, however, only when the steady-state specifications do not lead to the imposition of the Bode gain of the loop gain transfer function. The transfer function of a PI controller is

$$
C_{\mathrm{PI}}(s)=K_{p}\left(1+\frac{1}{T_{i} s}\right)=K_{p} \frac{T_{i} s+1}{T_{i} s} .
$$

By defining $\tilde{G}(s)=G(s) / s$, it is found

$$
\begin{aligned}
M_{g} & =\frac{1}{\left|\tilde{G}\left(j \omega_{g}\right)\right|}=\frac{\omega_{g}}{\left|G\left(j \omega_{g}\right)\right|} \\
\varphi_{g} & =\mathrm{PM}-\pi-\arg \tilde{G}\left(j \omega_{g}\right)=\mathrm{PM}-\frac{\pi}{2}-\arg G\left(j \omega_{g}\right) .
\end{aligned}
$$

To find the parameters of the PI controller, equation

$$
M_{g} e^{j \varphi_{g}}=K_{p} \frac{j T_{i} \omega_{g}+1}{T_{i}}
$$

must be solved in $K_{p}>0$ and $T_{i}>0$. 


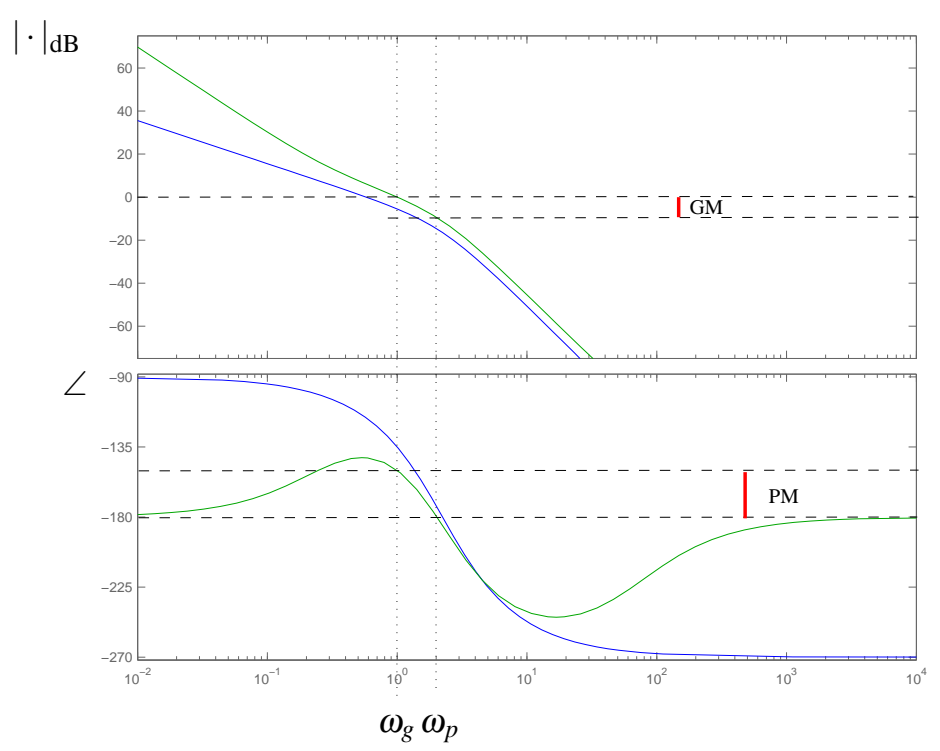

Figure 5: Bode diagram of the plant $G$ (blue solid line) and of the loop gain transfer function $L$ (green solid line). It can be seen that the desired specifications $\mathrm{PM}=30^{\circ}, \mathrm{GM}=3=9.54 \mathrm{~dB}$ and $\omega_{g}=1 \mathrm{rad} / \mathrm{s}$ are all satisfied.

Theorem 5.1 Equation (46) admits solutions in $K_{p}>0$ and $T_{i}>0$ if and only if

$$
\varphi_{g} \in\left(0, \frac{\pi}{2}\right)
$$

If (47) is satisfied, the solution of (46) is given by

$$
\begin{aligned}
K_{p} & =\frac{M_{g} \sin \varphi_{g}}{\omega_{g}} \\
T_{i} & =\frac{1}{\omega_{g}} \tan \varphi_{g} .
\end{aligned}
$$

As already observed, when the steady-state requirements lead to the imposition of the ratio $K_{p} / T_{i}$, the problem of assigning the phase margin and the crossover frequency of the loop gain transfer function cannot be solved in general. Indeed, if we define $\tilde{G}(s)=\frac{K_{p}}{T_{i} s} G(s)$ and $\tilde{C}_{\mathrm{PI}}(s)=1+T_{i} s$, the values $M_{g}=1 /\left|\tilde{G}\left(j \omega_{g}\right)\right|$ and $\varphi_{g}=\mathrm{PM}-\pi-\arg \tilde{G}\left(j \omega_{g}\right)$ are such that the identity $1+j \omega_{g} T_{i}=M_{g} e^{j \varphi_{g}}$ must be satisfied. The latter yields $M_{g} \cos \varphi_{g}=1$ and $M_{g} \sin \varphi_{g}=\omega_{g} T_{i}$, which are two equations in one unknown $T_{i}$; the first does not even depend on $T_{i}$, but only on the transfer function of the plant $G(s)$. As such, the problem at hand now admits solutions only if the equality constraint $M_{g} \cos \varphi_{g}=1$ is satisfied. If that constraint is satisfied, $T_{i}=M_{g} \sin \varphi_{g} / \omega_{g}$.

Example 5.1 Consider the plant described by the transfer function in Example 4.1. Find the parameters of a PI controller that achieves a phase margin of $45^{\circ}$, a crossover frequency $\omega_{g}=10 \mathrm{rad} / \mathrm{s}$ and zero velocity error. First, let us compute $M_{g}=1 /|G(j)|=20 \sqrt{26}$ and $\varphi_{g}=\mathrm{PM}-\pi-\arg G(j)=-\pi / 4+\arctan (1 / 2)$. The angle 
$\varphi_{g}$ lies in $(0, \pi / 2)$, so that a PI controller meeting the specifications exists. The parameters can be found with

$$
\begin{aligned}
K_{p} & =\frac{M_{g} \sin \varphi_{g}}{\omega_{g}}=40 \sqrt{2}, \\
T_{i} & =\frac{1}{\omega_{g}} \tan \varphi_{g}=\frac{1}{15} .
\end{aligned}
$$

The transfer function of the PI controller is

$$
C_{\mathrm{PI}}(s)=\frac{M_{g} \omega_{g} \cos \varphi_{g}+M_{g} \sin \varphi_{g} s}{\omega_{g} s}=40 \sqrt{2} \frac{s+15}{s} .
$$

Notice that, since in this case we have $M_{g} \cos \varphi_{g}=\sqrt{3} \cos (-\pi / 4+\arctan 1 / 2) \neq 1$, we cannot solve the problem with constraints on $K_{i}$.

\section{PD controllers}

As for the design of PI controllers, the synthesis techniques presented for the imposition of phase margin and crossover frequency of the loop gain transfer function can be used for PD controllers only when the steadystate specifications do not lead to the imposition of the proportional sensitivity $K_{p}$. The transfer function of a PD controller is

$$
C_{\mathrm{PD}}(s)=K_{p}\left(1+T_{d} s\right)
$$

To find the parameters of the PD controller, the equation

$$
M_{g} e^{j \varphi_{g}}=K_{p}\left(1+j T_{d} \omega_{g}\right)
$$

must be solved in $K_{p}>0$ and $T_{d}>0$.

Theorem 6.1 Equation (48) admits solutions in $K_{p}>0$ and $T_{d}>0$ if and only if

$$
\varphi_{g} \in\left(0, \frac{\pi}{2}\right)
$$

If (49) is satisfied, the solution of (48) is given by

$$
\begin{aligned}
K_{p} & =M_{g} \cos \varphi_{g} \\
T_{d} & =\frac{1}{\omega_{g}} \tan \varphi_{g}
\end{aligned}
$$

When the imposition of the steady-state specifications lead to a sharp constraint on $K_{p}$, and define $\tilde{G}(s)=$ $K_{p} G(s)$ and $\tilde{C}_{\mathrm{PD}}(s)=1+T_{d} s$, the values $M_{g}=1 /\left|\tilde{G}\left(j \omega_{g}\right)\right|$ and $\varphi_{g}=\mathrm{PM}-\pi-\arg \tilde{G}\left(j \omega_{g}\right)$ lead to the identity $1+j \omega_{g} T_{d}=M_{g} e^{j \varphi_{g}}$, which in turn leads to the two equations $M_{g} \cos \varphi_{g}=1$ and $M_{g} \sin \varphi_{g}=\omega_{g} T_{d}$, so that the problem admits solutions only if the constraint $M_{g} \cos \varphi_{g}=1$ is satisfied. In that case, $T_{d}=M_{g} \sin \varphi_{g} / \omega_{g}$. 


\section{Proper PD controllers}

Now we consider an alternative form of the PD controller, described by the transfer function:

$$
C_{\mathrm{PD}}^{\prime}(s)=K_{p}\left(1+\frac{T_{d} s}{1+\tau_{d} s}\right)=K_{p} \frac{1+\left(T_{d}+\tau_{d}\right) s}{1+\tau_{d} s},
$$

with $K_{p}, T_{d}, \tau_{d}>0$. In this case, the proposed method can be applied even in the presence of steady-state requirements on the position error for type- 0 plants, on the velocity error for type- 1 plants, and on the acceleration error for type-2 plants. In fact, the transfer function $C_{\mathrm{PD}}^{\prime}(s)$ is exactly that of a lead network. Since the transfer function $C_{\mathrm{PD}}^{\prime}(s)$ does not contain poles at the origin, usually the steady-state specifications lead to constraints on the proportional sensitivity $K_{p}$. In that case, let us define $\tilde{G}(s)=K_{p} G(s)$, and

$$
\tilde{C}_{\mathrm{PD}}^{\prime}(s)=\frac{1+\left(T_{d}+\tau_{d}\right) s}{1+\tau_{d} s}
$$

If we also set $\tilde{C}_{\mathrm{PD}}^{\prime}(j \omega)=M(\omega) e^{j \varphi(\omega)}$, by imposing $\left|L\left(j \omega_{g}\right)\right|=1$ and $\mathrm{PM}=\pi+\arg L\left(j \omega_{g}\right)$, we find that, as before, in order to find the parameters of the controller, equation

$$
M_{g} e^{j \varphi_{g}}=\frac{1+j\left(T_{d}+\tau_{d}\right) \omega_{g}}{1+j \tau_{d} \omega_{g}}
$$

must be solved in $T_{d}, \tau_{d}>0$.

Theorem 7.1 Equation (51) admits solutions $T_{d}, \tau_{d}>0$ if and only if

$$
M_{g}>1, \quad \varphi_{g} \in\left(0, \frac{\pi}{2}\right), \quad M_{g}>\frac{1}{\cos \varphi_{g}} .
$$

If (52) are satisfied, the solution of (51) is given by

$$
\begin{aligned}
T_{d} & =\frac{M_{g}^{2}-2 M_{g} \cos \varphi_{g}+1}{M_{g} \omega_{g} \sin \varphi_{g}} \\
\tau_{d} & =\frac{M_{g} \cos \varphi_{g}-1}{M_{g} \omega_{g} \sin \varphi_{g}}
\end{aligned}
$$

\section{Graphical and approximate solution to the design problem}

The synthesis methods developed in the previous sections are based on closed-form formulae for the computation of the parameters of the PID controller. In this regard, it could be argued that often a full knowledge of the dynamics of the plant is not available in practice and therefore the presented closed-form formulae are of little interest. On the contrary, the approach presented here still offers a solution even when the model of the plant is not exactly known, which is may be based on (i) graphical considerations similar in spirit to those considered in the literature [16] (but that can be carried out on any of the standard diagrams for the frequency response), (ii) on the approximation of the plant with a first or second order transfer function [7], or even (iii) on the results of an experiment conducted on the plant in the same spirit of the Ziegler and Nichols methods, [18]. In this and in the following section we discuss these issues. 
As already mentioned, a graphical version of the method presented in this paper can implemented using any of the frequency domain plots usually employed in control to represent the frequency response dynamics, i.e., Bode diagrams, Nyquist diagrams or Nichols charts. This is due to the fact that the formulae used to derive the parameters of the PID controller are expressed in terms of the magnitude and the argument of the frequency response of the plant at a given crossover frequency, which is readable over any of these diagrams.

Example 8.1 Consider Example 5.12 in [6], in which the control of a small airplane has to be implemented via a PID controller achieving a crossover frequency of $8 \mathrm{rad} / \mathrm{s}$, a phase margin of $75^{\circ}$ and a ratio $T_{i} / T_{d}$ equal to 4. The Nyquist plot of the plant, in which the input is the pitch attitude and the output is the elevator angle, both expressed in degrees, is represented by the blue line in Figure 6. Given the point of the Nyquist plot

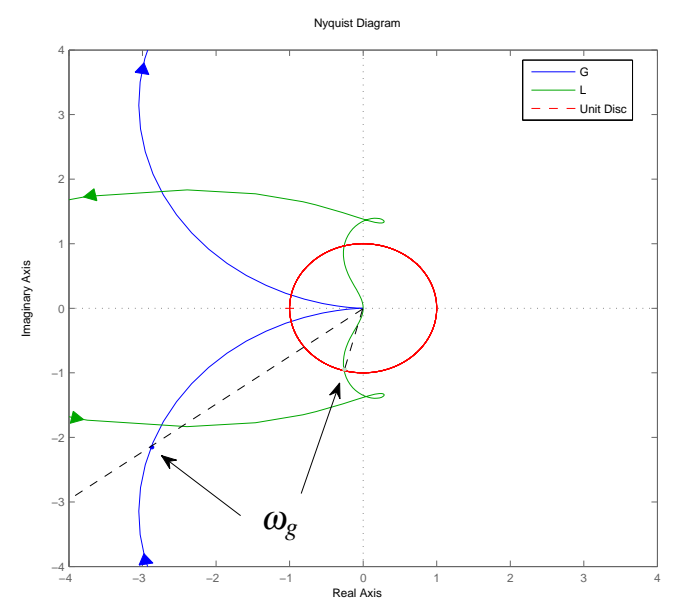

Figure 6: Nyquist diagram of the plant $G$ (blue solid line) and of the loop gain transfer function $L$ (green solid line).

corresponding to the desired gain crossover frequency $\omega_{g}=8 \mathrm{rad} / \mathrm{s}$, i.e.,

$$
G\left(j \omega_{g}\right) \simeq-2.9-2.2 j
$$

we can estimate

$$
\begin{aligned}
M_{g} & =\frac{1}{\sqrt{2.9^{2}+2.2^{2}}}=0.2747, \\
\varphi_{g} & =\mathrm{PM}-\pi-\arctan \frac{2.2}{2.9}-\pi=0.6600 .
\end{aligned}
$$

These values lead to the parameters

$$
K_{p}=0.2170, \quad T_{i}=0.5105 \mathrm{~s}, \quad T_{d}=0.1276 \mathrm{~s} .
$$

The frequency response of the loop gain transfer function obtained by combining the PID controller thus obtained with the transfer function of the plant given in [6], i.e.,

$$
G(s)=160 \frac{(s+2.5)(2+0.7)}{\left(s^{2}+5 s+40\right)\left(s^{2}+0.03 s+0.06\right)}
$$


is depicted with the green solid line in Figure 6. By direct inspection it can be seen that the loop gain transfer function guarantees the desired phase margin and crossover frequency. And in fact, using the MATLAB ${ }^{\circledR}$ routine margin on the loop gain given by the series of the PID controller with (53) and $G(s)$ shows that the phase margin and gain crossover frequency obtained are $74.93^{\circ}$ and $7.96 \mathrm{rad} / \mathrm{s}$, respectively. The step response of the controlled system is given in Figure 7.

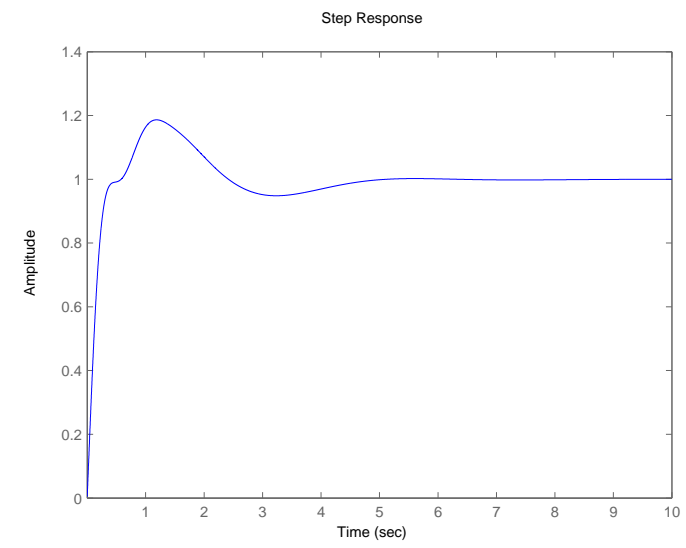

Figure 7: Step response of the controlled system.

The approach described in the previous example may be employed even in absence of graphical descriptions of the plant transfer function. Indeed, in the very same spirit of the Ziegler and Nichols method, we may "perform an experiment" on the plant by feeding it with a sinusoidal input with frequency $\omega_{g}$, i.e. the desired crossover frequency. From the steady-state output we can estimate $G\left(j \omega_{g}\right)$, and hence $M_{g}$ and $\varphi_{g}$ and we can thus readily apply the proposed method.

\section{Second order plus delay approximation}

Several tuning techniques proposed in the literature do not require exact knowledge of the mathematical model of the plant, but rely on its first or second order plus delay approximation, [7, 2, 3]. While in this paper the formulae for the parameters of the PID controller have been obtained under the assumption of exact knowledge of the transfer function of the plant, the procedure outlined can be used in conjuction with the heuristics or numerical methods based on these approximations. In this section we show that the formulae presented in this paper can be specialised to the case of a second order plus delay approximation of the plant dynamics, and compare our results with those in [7], in the case of specifications on the stability margins and gain crossover frequency. We consider the second order plus delay model

$$
G(s)=\frac{K}{\left(1+\tau_{1} s\right)\left(1+\tau_{2} s\right)} e^{-T s}
$$


where $\tau_{1}, \tau_{2}, T>0$ and $K>0$. A direct calculation shows that

$$
\begin{aligned}
M_{g} & =\frac{\sqrt{\left(1+\omega_{g}^{2} \tau_{1}^{2}\right)\left(1+\omega_{g}^{2} \tau_{1}^{2}\right)}}{K}, \\
\varphi_{g} & =\theta-\pi+\arctan \left(\omega_{g} \tau_{1}\right)+\arctan \left(\omega_{g} \tau_{2}\right),
\end{aligned}
$$

where $\theta=\mathrm{PM}+\omega_{g} T$, which lead to

$$
M_{g} \cos \left(\varphi_{g}\right)=\frac{\omega_{g}\left(\tau_{1}+\tau_{2}\right) \sin \theta-\left(1-\omega_{g}^{2} \tau_{1} \tau_{2}\right) \cos \theta}{K}
$$

If the specifications are on the ratio $\sigma=T_{d} / T_{i}$, the parameters of the compensator can be computed using directly (12-14), that with this particular model yield

$$
\begin{aligned}
K_{p} & =\frac{\omega_{g}\left(\tau_{1}+\tau_{2}\right) \sin \theta-\left(1-\omega_{g}^{2} \tau_{1} \tau_{2}\right) \cos \theta}{K}, \\
T_{i} & =\frac{\left(1-\omega_{p}^{2} \tau_{1} \tau_{2}\right) \tan \theta+\omega_{p}\left(\tau_{1}+\tau_{2}\right)}{\left(1-\omega_{p}^{2} \tau_{1} \tau_{2}\right)-\omega_{p}\left(\tau_{1}+\tau_{2}\right) \tan \theta}, \\
T_{d} & =T_{i} \sigma .
\end{aligned}
$$

If the specifications are on both the phase and gain margin (and on the gain crossover frequency), we must also compute

$$
\begin{aligned}
M_{p} & =\frac{\sqrt{\left(1+\omega_{p}^{2} \tau_{1}^{2}\right)\left(1+\omega_{p}^{2} \tau_{1}^{2}\right)}}{K}, \\
\varphi_{p} & =-\pi+\omega_{p} T+\arctan \left(\omega_{p} \tau_{1}\right)+\arctan \left(\omega_{p} \tau_{2}\right),
\end{aligned}
$$

so that

$$
M_{p} \cos \left(\varphi_{p}\right)=\frac{\omega_{p}\left(\tau_{1}+\tau_{2}\right) \sin \left(\omega_{p} T\right)-\left(1-\omega_{p}^{2} \tau_{1} \tau_{2}\right) \cos \left(\omega_{p} T\right)}{\mathrm{GM} \cdot K}
$$

Therefore, in order to achieve the desired phase and gain margins at the desired gain crossover frequency, we need to find the roots $\omega_{p}$ of the equation

$$
\psi\left(\omega_{p}\right)=\omega_{p}\left(\tau_{1}+\tau_{2}\right) \sin \left(\omega_{p} T\right)-\left(1-\omega_{p}^{2} \tau_{1} \tau_{2}\right) \cos \left(\omega_{p} T\right)-\mathrm{GM} \cdot K \cdot M_{g} \cos \left(\varphi_{g}\right)=0 .
$$

Notice that this time function $\psi\left(\omega_{p}\right)$ is not polynomial, since the transfer function of the model is not rational. The roots of this equation can be determined numerically, as shown in the following example. Of all the roots of $\psi\left(\omega_{p}\right)$, one satisfying the conditions of Theorem 3.2 must be determined (if no such roots exist, the problem does not admit solutions), and compute the parameters of the PID controller using (26-28).

Example 9.1 In this example we compare the results presented in this paper with those based on a second order approximation described in [7]. In particular, we consider the process in [7, Table 5] described by the transfer function $1 /(s+1)^{5}$ and approximated with the second order model

$$
G(s)=\frac{e^{-1.73 s}}{(1+1.89 s)^{2}} .
$$




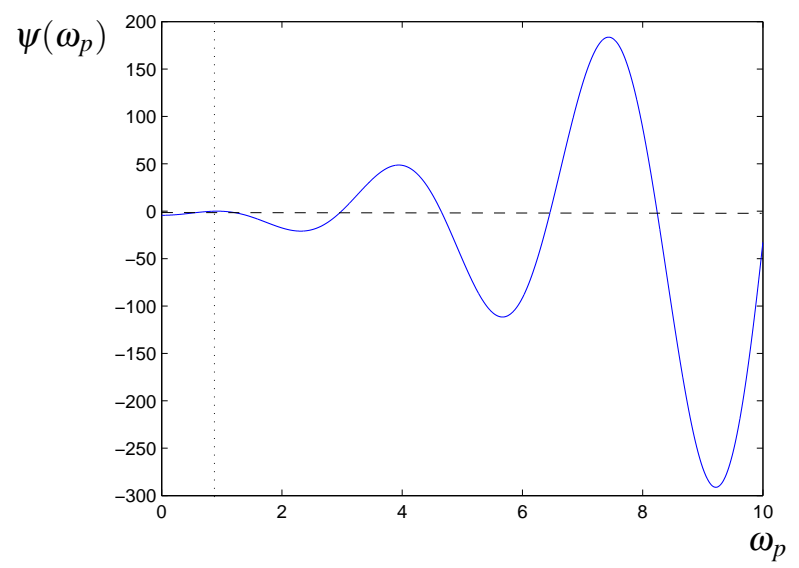

Figure 8: Function $\psi\left(\omega_{p}\right)$.

The design specifications considered are on the phase and on the gain margin, which are required to be equal to $60^{\circ}$ and 3 , respectively. The desired gain crossover frequency is $0.30 \mathrm{rad} / \mathrm{s}$. This problem can be solved as described above with $\tau_{1}=\tau_{2}=1.89 \mathrm{~s}, T=1.73 \mathrm{~s}$ and $K=1$. In this case, function $\psi\left(\omega_{p}\right)$ is depicted in Figure 8. It can be numerically established that the smallest root of $\psi\left(\omega_{p}\right)$ is at $\omega_{p}=0.8728 \mathrm{rad} / \mathrm{s}$. It is a matter of direct substitution to see that this frequency satisfies the conditions given in Theorem 3.2. This means that the control problem is solvable, and we can use $\omega_{p}$ to compute the parameters of the compensator:

$$
K_{p}=1.1038, \quad T_{i}=3.7797 \mathrm{~s}, \quad \mathrm{~T}_{\mathrm{d}}=0.8291 \mathrm{~s} .
$$

With these values, the phase and gain margins of the loop gain transfer function obtained by the series of the real process and the PID controller designed using its second-order approximation are $62.26^{\circ}$ and 3.26 , respectively, and the real gain crossover frequency is at $0.3173 \mathrm{rad} / \mathrm{s} .{ }^{1}$

The closed-form formulae given in this paper for the parameters of the PID controller are given in finite terms. Hence, a remarkable advantage of this method is the fact that these formulae can be applied to any plant approximation, even though in this section for the sake of comparison with the existing methods only the second order plus delay approximation has been considered. As such, the flexibility offered by the method presented here also extends to the case where the transfer function of the plant to be controlled is not exactly known, and necessarily guarantees a better performance when a better approximation of the plant dynamics is available. This also means that the method proposed in this paper outperformes any method constructed upon a plant approximation with a fixed structure.

\footnotetext{
${ }^{1}$ The values of the parameters of the PID controller are different from those obtained here because in [7] the different transfer function for the PID controller

$$
C_{\mathrm{PID}}(s)=K_{P} \frac{\left(1+T_{i} s\right)\left(1+T_{d} s\right)}{T_{i} s}
$$

is utilised. The values of the phase margin and gain margin obtained in [7] are $62.5^{\circ}$ and 3.38 , respectively.
} 


\section{Conclusions}

A unified approach has been presented that enable the parameters of PID, PI and PD controllers (with corresponding approximations of the derivative action when needed) to be computed in finite terms given appropriate specifications expressed in terms of steady-state performance, phase/gain margins and gain crossover frequency. The synthesis tools developed in this paper eliminate the need of trial-and-error and heuristic procedures in frequency-response design, and therefore they undoubtedly outperforms the heuristic, trial-and-error and graphic approaches proposed so far in the literature for the feedback control problem with specifications on the steady-state performance and on the gain/phase margins, in the case of perfect knowledge of the model of the plant. When the plant model is not exactly known - as is often the case in practice - the present method can still be fruitfully employed for the design of the PID controller. Indeed, the formulae delivering the controller parameters only require the magnitude and the argument of the frequency response of the plant at the desired crossover frequency. These data can be obtained graphically by direct inspection over any of the Nyquist, Bode and Nichols diagrams (with the advantage that no special charts are needed). Alternatively, the closed-form design techniques can be used jointly with a first or second order plus delay approximation of the plant to deliver the desired values of the stability margins and crossover frequency.

\section{References}

[1] Charles L. Phillips. Analytical Bode Design of Controllers. IEEE Transactions on Education, E-28, no. 1 , pp. $43-44,1985$.

[2] K.J. Åström, and T. Hagglund, PID Controllers: Theory, Design, and Tuning, second edition. Instrument Society of America, Research Triangle Park, NC, 1995.

[3] K.J. Åström, and T. Hagglund, Advanced PID Control. Instrument Society of America, Research Triangle Park, NC, 2006.

[4] A. Datta, M.-T. Ho, and S.P. Bhattacharyya. Structure and Synthesis of PID Controllers. Springer, 2000.

[5] A. Ferrante, A. Lepschy, and U. Viaro. Introduzione ai Controlli Automatici. UTET Universit, 2000.

[6] G. Franklin, J.D. Powell, and A. Emami-Naeini . Feedback Control of Dynamic Systems. Prentice Hall, 2006.

[7] W.K. Ho, and C.C. Hang, and L.S. Cao, Tuning of PID Controllers Based on Gain and Phase Margin Specifications. Automatica, vol. 31, no. 3, pp. 497-502, 1995.

[8] L.H. Keel and S.P. Bhattacharyya. Controller Synthesis Free of Analytical Models: Three Term Controllers. IEEE Transactions on Automatic Control, AC-53, no. 6, pp. 1353-1369, 2008. 
[9] K. Kim, and Y.C. Kim,, The Complete Set of PID Controllers with Guaranteed Gain and Phase Margins. Proceedings of the $44^{\text {th }}$ IEEE Conference on Decision and Control, and the European Control Conference 2005, pp. 6533-6538, Seville, Spain, December 12-15, 2005.

[10] G. Marro, TFI: Insegnare ed apprendere i controlli automatici di base con MATLAB ${ }^{\circledR}$. Zanichelli Ed., Bologna (Italy), 1998.

[11] K. Ogata. Modern Control Engineering. 2nd edn. Prentice Hall, Englewood Cliffs, NJ, 2000.

[12] S. Skogestad. Simple analytic rules for model reduction and PID controller tuning. Journal of Process Control, vol. 13, pp. 291-309, 2003.

[13] A. Visioli, Practical PID Control. Advances in Industrial Control, Springer-Verlag, 2006.

[14] W.R. Wakeland. Bode Compensation Design. IEEE Transactions on Automatic Control, AC-21, no. 5, pp. 771-773, 1976.

[15] R. Zanasi and G. Marro, New formulae and graphics for compensator design. Proceedings of the 1998 IEEE International Conference on Control Applications, Vol. 1, pp. 129-133, 1-4 September 1998.

[16] K.S. Yeung, and K.H. Lee. A Universal Design Chart for Linear Time-Invariant Continuous-Time and Discrete-Time Compensators. IEEE Transactions on Education, E-43, no. 3, pp. 309-315, 2000.

[17] R. Zanasi and R. Morselli, Discrete Inversion Formulas for the Design of Lead and Lag Discrete Compensators. Proceedings of the European Control Conference 2009, pp. 5069-5074, August 23-26, 2009.

[18] J.G. Ziegler, and N.B. Nichols, Optimum settings for automatic controllers. Trans. ASME, vol. 64, pp. 759-768, 1942. 